\title{
Modeling the Heat Resistance of Nickel-based Superalloys by a Deep Learning Neural Network
}

\author{
Dmitry A. Tarasov ${ }^{1, a)}$, Andrey G. Tyagunov ${ }^{1, b)}$, Oleg B. Milder ${ }^{1, c)}$ \\ ${ }^{1}$ Ural Federal University, Mira str., 19, Ekaterinburg, RUSSIA 620002 \\ ${ }^{a)}$ Corresponding author: datarasov@yandex.ru \\ b)adi8@yandex.ru \\ c)milder@mail.ru
}

\begin{abstract}
The nickel-based superalloys are unique materials with complex alloying used in the manufacture of gas turbine engines. The alloys exhibit excellent resistance to mechanical and chemical degradation under the high loads and longterm isothermal exposures. The main service property of the alloy is its heat resistance, which is expressed by the tensile strength. Simulation of changes in the heat resistance is an important engineering problem, which would significantly simplify the design of new alloys. In this paper, we apply a deep learning neural network to predict the tensile strength values and to compare the predictive ability of the proposed approach. Also, the results are presented of the feed-forward neural network accounting changes in heat resistance $v s$ isothermal exposures that are expressed in the complex LarsonMiller parameter.
\end{abstract}

Keywords: Nickel-based superalloys, Artificial neural networks, Deep learning, Simulation, Tensile strength, LarsonMiller parameter.

\section{INTRODUCTION}

The nickel-based superalloys is a family of special heat resistant alloys consisting the several hundred varieties; moreover, their number is constantly growing. They are widely applied in manufacturing the gas turbine engines' parts. The matrix of the alloys is formed by nickel as an isomorphic material. The doping is formed by many other elements [1]. The major service properties of the alloys are the heat resistance and structural thermal stability. The heat resistance is the ability of material to resist the load at elevated temperatures without undergoing permanent deformation or fracture. The thermal stability is the ability of a material to retain properties during a period of prolonged isothermal exposures [2]. To determine the heat resistance, mechanical tests on long-term tensile strength are carried out. This variable shows the greatest mechanical stress that the material could resist without breaking at a given temperature, exposure time, and working conditions. The magnitude of the tensile strength is denoted as $\sigma_{t}^{\tau}$, where $\tau$ is the isothermal exposure time (in hours), and $t$ is the temperature $\left(\right.$ in ${ }^{\circ} \mathrm{C}$ ).

The unique properties of materials are determined, firstly, by the careful selection of the chemical composition, and secondly, by applying the single-crystal casting. However, improving the properties of the alloyed requires the use of expensive additives for doping, which leads to a significant increase in production costs. The duration and high cost of physical experiments on alloys also have a significant contribution to the price of the materials. For civil applications this is vital. Thus, development of new superalloys grades that both relatively cheap and maintaining the desired performance are substantial engineering tasks.

Since the physical modeling of the alloys are time consuming and quite expensive, the mathematical simulation might be a method that would simplify the design and development of new materials. The key problem in modeling is the fact that the alloys are tested in fairly narrow time and temperature ranges that leads to the insufficient volume of an initial database for building the statistical models. Nevertheless, novel computational methods like the artificial neural networks (ANN) made it possible to extrapolate the results of tests for the long-term strengths without 
implementation of the expensive and long-term full-scale experiments. The use of neural networks is suitable for modeling correlations that are blurry, difficult to describe, or which cannot be accurately simulated by the physical models. Since artificial modeling of the neural networks is a non-linear statistical method, it can be used to solve problems that are not amenable to solution by the conventional statistical methods [3]. This is the major reason for applying the neural modeling in such a complicated task like superalloys properties simulation.

Previously, the ANN were used to analyze the nickel-based alloys [4, 5]. However, the major goal of these works was to synthesize new chemical compositions of the alloys [4]. There are, also, the ANN applications to model change in the coefficient of thermal expansion, to model energy hysteresis, to predict a low-cycle fatigue energy, to model the development of fatigue cracks, to predict the occurrence of material defects, and to model the time to failure.

It did not succeeded to find works devoted to calculation of the heat resistance on the basis of establishing the following important relation. Namely, one between the alloy chemical composition and the experimental values of the long-term tensile strength. Also, there are no works with analysis of the changes in the heat resistance and thermal stability of a whole number of alloys.

The experiments mentioned above were based on utilization of different types of the common feed-forward artificial neural networks with the standard training procedures. The bayesian regularized artificial neural networks (BRANN) are more robust than the standard back-propagation ones and might reduce or even eliminate the need for cross-validation helping to shorten the experiment time. They have shown satisfactory predictive ability along with the resistance to overtraining during application [6]. Accounting that, we have previously predicted service properties of the superalloys in connection with their chemical composition [7]. However, not all chemical elements have the same effect on the properties of alloys. This should be considered while a network is built. However, the common feed-forward approach do not able to imply such a complication of the model. Therefore, we must apply a more flexible framework.

In this work, using the same database as in [7], we take a step towards the use of a deep learning network, in which each layer is responsible for a specific set of the service properties. We will carry out the similar procedure for predicting the properties of alloys and compare the approaches of the BRANN and the deep learning network.

\section{APPROACH AND EXPERIMENTAL}

The collected alloys database contains the test information and service properties on 308 nickel-based superalloys. In total, 47 pairs $(\tau, t)$ were taken into account. The samples include 23 alloying elements: $\mathrm{C}, \mathrm{Cr}, \mathrm{Co}, \mathrm{Mo}, \mathrm{W}, \mathrm{Al}, \mathrm{Ti}$, $\mathrm{Nb}, \mathrm{B}, \mathrm{Fe}, \mathrm{Y}, \mathrm{Zr}, \mathrm{Ta}, \mathrm{Re}, \mathrm{Ru}, \mathrm{V}, \mathrm{Ce}, \mathrm{La}, \mathrm{S}, \mathrm{Si}, \mathrm{Mn}, \mathrm{P}, \mathrm{Hf}$, and Ni. For each alloy in the database, no more than 25 test results $\left(\sigma_{t}^{\tau}\right)$ were known. For training the networks, records on alloys with at least 5 known values $\sigma_{t}^{\tau}$ were selected. Hence, the training set has been formed by 167 alloys. Each alloy forms as many samples for the network training as many different physical tests were conducted (i.e. there is a relevant information).

The main task of the computational experiment was to fill in the missing $\sigma_{t}^{\tau}$ values in the database and to compare the prediction ability of two frameworks. We predict unknown $\sigma_{t}^{\tau}$ and evaluate the prediction precision. For verification by calculating the error between the real and predicted values, we have extracted 9 randomly selected alloys from the database before the training. To create and train the networks, the MATLAB software with the builtin nntools was applied. To improve the accuracy of simulations, a specially developed input data pre-processing was carried out. The input parameters of the models are the chemical compositions of the alloys and their test time and temperature. Taking into account all possible alloying elements, the total number of input parameters was 25 . The models frameworks are shown in Fig. 1.

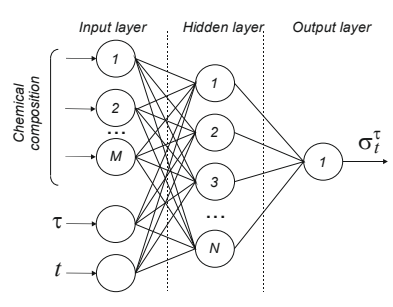

(a)

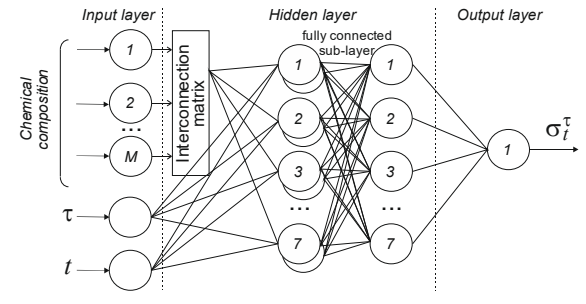

(b)

FIGURE 1. Models configuration: (a) a feed-forward network, (b) a deep learning network. 
The deep learning framework is based on the interconnection matrix that defines the linkages between the input layer and the first layer of hidden neurons. The matrix reflects the major metallurgical phase components forming a particular hidden layer. The verification sub-sample is shown in Table 1.

The framework's prediction ability is evaluated by the related root mean squared error (RRMSE) between the predictions and real data from the verification sub-sample and the whole database. Using the experimental and reconstructed values of the tensile strengths for the alloys from the sub-sample, we plotted the dependences of the heat resistance vs the Larson-Miller parameter.

TABLE 1. Chemical compositions of the verification sub-sample, wt $\%$ ( $\mathrm{Ni}$ is balansed).

\begin{tabular}{lllllllllllllll}
\hline Alloys & Cr & Co & Mo & W & Al & Ti & Ta & Nb & Re & Ru & Hf & C & B & Zr \\
\hline CMSX-10 & 2.0 & 3.0 & 0.4 & 5.0 & 5.7 & 0.2 & 8.0 & - & 6.0 & - & 0.03 & - & - & - \\
EPM-102 & 2.0 & 16.5 & 2.0 & 6.0 & 5.55 & - & 8.25 & - & 5.95 & 3.0 & 0.15 & 0.03 & - & - \\
IN100 & 10.0 & 15.0 & 3.0 & - & 5.5 & 4.7 & - & - & - & - & - & 0.18 & 0.014 & 0.06 \\
IN-713LC & 12.0 & - & 4.5 & - & 5.9 & 0.6 & - & 2.0 & - & - & - & 0.05 & 0.01 & 0.10 \\
IN-792 & 12.4 & 9.2 & 1.9 & 3.9 & 3.5 & 3.9 & 4.2 & - & - & - & - & 0.07 & 0.016 & 0.018 \\
Mar-M246 & 9.0 & 10.0 & 2.5 & 10.0 & 5.5 & 1.5 & 1.5 & - & - & - & 1.5 & 0.15 & 0.015 & 0.05 \\
MC-NG & 4.0 & - & 1.0 & 5.0 & 6.0 & 0.5 & 5.0 & - & 4.0 & 4.0 & 0.1 & - & - & - \\
TMS-138 & 2.9 & 5.9 & 2.9 & 5.9 & 5.9 & - & 5.6 & - & 4.9 & 2.0 & 0.1 & - & - & - \\
TMS-162 & 2.9 & 5.8 & 3.9 & 5.8 & 5.8 & - & 5.6 & - & 4.9 & 6.0 & 0.09 & - & - & - \\
\hline
\end{tabular}

\section{RESULTS AND DISCUSSION}

The BRANN in [30] contained 25 neurons in the input layer, 7 neurons in the hidden layer, and 1 output neuron. The errors (RRMSE) of the tensile strengths predictions distribution do not exceed 0.20 for most observations. The deep learning network after the training predicts at the same level of precision (RRMSE less than 0.19); however, it works rather faster and the application of the additional re-training procedure would lead to a better results.

Verification of the prediction accuracy of the completely trained model was carried out using a verification subsample. The result of the forecast was the values of the tensile strengths $\sigma_{t}^{\tau}$ corresponding to all possible sets of compositions and isothermal exposures of the sub-sample alloys. Based on the prediction, we have formed the tensile strengths vs $P_{L M}$ dependences for each of alloy from the verification sub-sample (see Fig. 2).

\section{CONCLUSION}

In this work, we have applied a deep learning neural network to prediction of the values of tensile strength of the nickel-based superalloys. The framework was rather complicated since each major metallurgical phase components formed a particular hidden layer, and the relations between inputs and first hidden layer were formed with the help of the specially developed interconnection matrix. The results of predictions were evaluated by the RRMSE between the known values of $\sigma_{t}^{\tau}$ from the sub-sample (nine randomly selected alloys) and their predicted values. The values of the errors of the preliminary BRANN simulation and of modeling by the deep learning network were nearly the same ( 0.20 and 0.19 respectively). However, both methods have their prospective and should be further developed.

Thus, a new approach to the analysis of the main service properties of the nickel-based superalloys and the calculation of the missing values of heat resistance for each alloy composition have been substantiated and tested by the independent verification sub-sample of nine alloys. The proposed method ensures the forecast accuracy that commensurate with the experimental error in measurement tests of the real alloys service properties. 

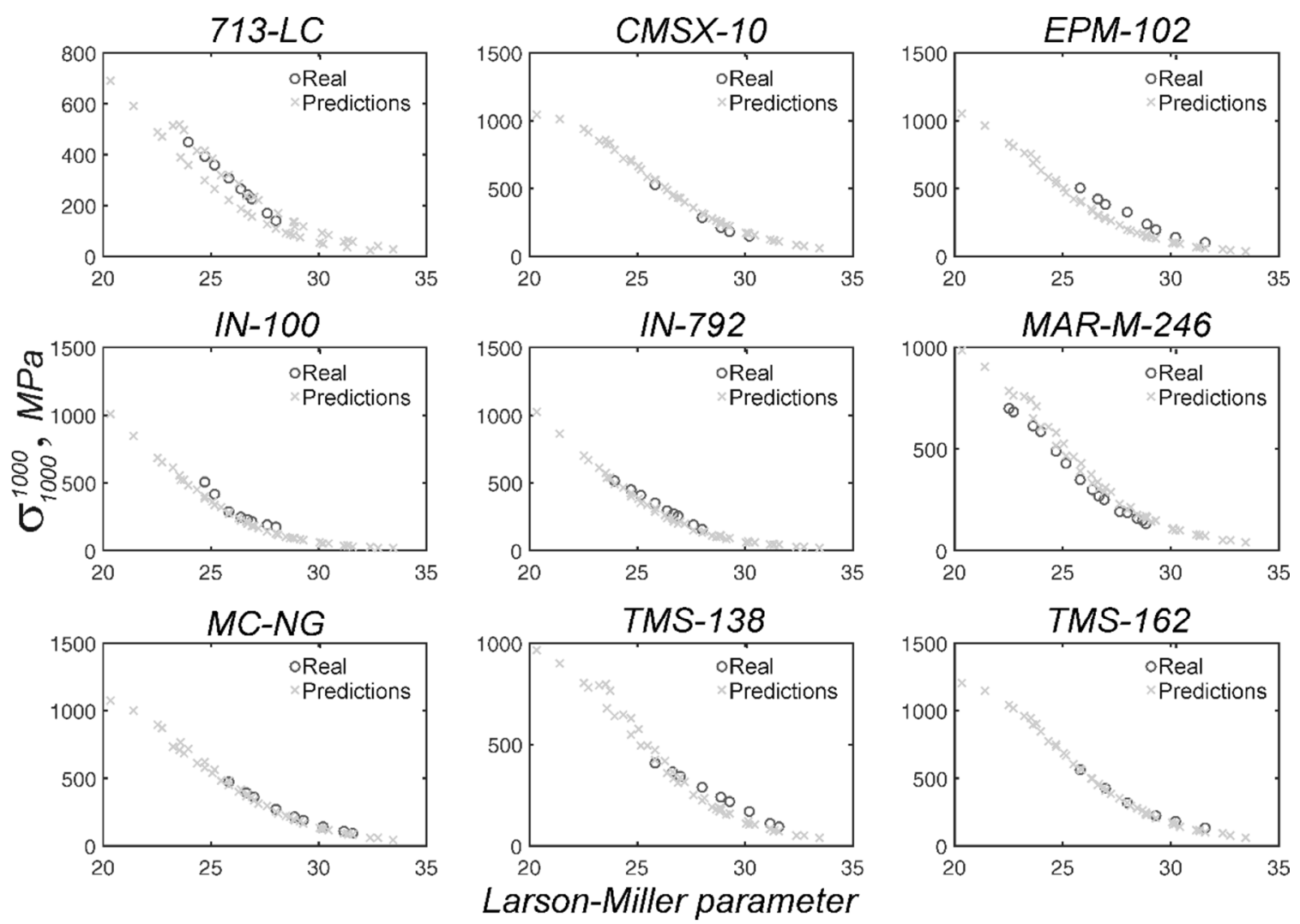

FIGURE 2. The model verification results for 9 common alloys that formed the sub-sample.

\section{REFERENCES}

1. V.P. Kuznetsov, V.P. Lesnikov, and N.A. Popov, "Structure and properties of single-crystal high-temperature nickel alloy", UrFU, 2004, 160p. (In Russian).

2. R.C. Reed "The Superalloys. Fundamentals and Applicatios", Cambridge University Press (2006), 372p.

3. S.O. Haykin "Neural Networks and Learning Machines", 3rd ed,, McMaster University, Ontario Canada (2009). 906p.

4. Y.S. Yoo, I.S. Kim, D.H. Kim, C.Y. Jo, H.M. Kim, C.N. Jone "The application of neural network to the development of single crystal superalloys, in Superalloys,. Ed. by K.A. Green (T.M. Pollock, H. Harada, T.E. Howson, R.C. Reed, J.J. Schirra, and S, Walston TMS (The Minerals, Metals \& Materials Society, 2004).

5. O.S. Nurgayanova and A.A. Ganeev, Polzunovsky almanac 3, 22-26 (2006). (In Russian).

6. F. Burden, D. Winkler "Bayesian Regularization of Neural Networks" in Artificial Neural Networks. Methods in Molecular Biology, 458. Livingstone D.J. (eds), Humana Press (2008)

7. A. Tyagunov, O. Milder, D. Tarasov "Application of Artificial Neural Networks for Prediction of Nickel-based Superalloys Service Properties Based on the Chemical Composition" in WSEAS Transactions on Environment and Development 15 (2019), pp. 113-119. 